\title{
Uso de microdebridador para el tratamiento del edema de Reinke
}

\author{
Use of microdebrider for Reinke's edema treatment
}

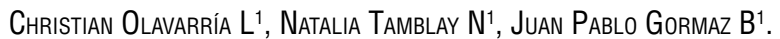

\begin{abstract}
RESUMEN
El edema de Reinke, corresponde al aumento de volumen y de consistencia del compartimento subepitelial, por lo cual la voz se hace de menor frecuencia y más áspera. El tratamiento de elección para esta patología consiste en la reducción quirúrgica de la matriz gelatinosa que se ubica bajo el epitelio. Se presenta aquí la técnica de reducción de esta matriz con microdebridador, lo cual permite dejar sin lesiones el epitelio cordal y lograr una cicatrización apropiada de la mucosa.
\end{abstract}

Palabras clave: Edema de Reinke, cuerda vocal.

\begin{abstract}
Reinke's edema is characterized by the swelling and increased consistency of the subepitelial compartment, which results in lower-than-normal pitch and hoarseness. Surgical reduction of the gelatinous matrix underlying the epithelium by means of a microdebrider is the choice treatment for this pathology, allowing for adequate healing of the mucosa, without damaging the vocal chord epithelium.
\end{abstract}

Key words: Reinke's edema, vocal chord epithelium.

\section{INTRODUCCIÓN}

El espacio de Reinke, corresponde al compartimiento subepitelial de las cuerdas vocales descrito por Reinke en el año 1895. Hirano catalogó este espacio como la lámina propia superficial y Zeitels lo identificó como la base para la vibración de las cuerdas vocales dadas sus características especiales de viscosidad. El aumento de volumen y de consistencia patológico de este espacio se denomina edema de Reinke, corditis polipoídea, laringitis polipoídea o degeneración polipoídea. El edema de Reinke produciría por una lesión y transudado de los capilares subepiteliales debido a múltiples factores irritantes, produciendo una distorsión de las cualidades vibratorias de este componente de la cuerda vocal. Los factores de riesgo para desarrollar esta patología son el sexo femenino, el tabaquismo, el abuso vocal y el reflujo faringo-laríngeo ${ }^{1}$.

El edema de Reinke se presenta clínicamente como un cambio gradual de la voz, la que se hace más áspera y de menor frecuencia, cambio que es más notorio en las mujeres. El edema de las cuerdas vocales habitualmente es bilateral, pero a menudo asimétrico y generalmente no se acompaña de síntomas respiratorios. En la estroboscopía la onda mucosa puede ser hiperdinámica 0 bien de pobre flexibilidad por el efecto de masa. En ocasiones el edema de Reinke puede ocultar una lesión maligna'.

El tratamiento de esta patología comienza con la eliminación de los factores de riesgo como suspender el tabaquismo y manejar el reflujo. El pilar del tratamiento en los casos más avanzados es la cirugía la que puede ser:

- Decorticación clásica: presenta largos periodos de recuperación de la voz después de la cirugía, produciendo cicatrices y adherencias en el subepitelio que pueden derivar en una voz permanentemente alterada ${ }^{2}$.

- Reducción de la matriz gelatinosa de la lámina propia superficial, descrita por Hirano: se realiza una reducción controlada de la lámina propia superficial, dejando indemne el ligamento vocal ${ }^{3}$.

Si bien en la teoría, resulta evidente la importancia de preservar la mayor cantidad de epitelio de la cuerda vocal, en la práctica resulta bastante difícil, llegando finalmente a la decorticación involuntaria durante el procedimiento. Esto se hace particularmente patente cuando el contenido del espacio de Reinke es más gelatinoso que líquido y no es posible de aspirar 0 liberar con facilidad.

1 Médico Servicio de Otorrinolaringología, Hospital Clínico Universidad de Chile. 
Recientemente se han descrito técnicas para ayudar al cirujano en la preservación de la mucosa como la utilización del láser $\mathrm{CO}_{2}^{4,5}$ para la incisión de la mucosa o la succión del edema con microdebridador $^{6}$, técnica que describiremos.

\section{TÉCNICA QUIRÚRGICA}

Se realiza una adecuada exposición de la zona operatoria, a través de laringoscopía directa y microscopio (Figura 1).

Se realiza una incisión en la cara superior de la cuerda vocal con microtijera o microbisturí, levantando un microflap lateral con un elevador disector. Mientras el microflap es sostenido hacia medial con una pinza Bouchayer ${ }^{6}$, el mixioma de la lámina superficial es succionado con un microdebridador con una hoja laríngea biselada de 2,9 mm de diámetro, cuidando de dejar una lámina propia de tamaño adecuado sin romper el epitelio subyacente (Figura 2).

Para óptimos resultados el microdebridador debe utilizarse con una baja presión de succión y una velocidad de rotación media (2.500 a 3.000 rpm). Luego, el microflap se repone retirando el exceso de mucosa con microtijera ${ }^{6}$.

Se le indica al paciente reposo vocal estricto por 7 días. Se evalúa con telelaringoscopía a la semana posoperado. Posteriormente debe recibir tratamiento fonoaudiológico, ya que se debe reeducar al paciente para que no fone con una presión subglótica aumentada ${ }^{1}$.

Los inconvenientes de esta técnica son que requiere instrumental adecuado para manejar el flap de mucosa con delicadeza y el costo de la punta laríngea para el microdebridador. Sin embargo, los beneficios en reducir la cicatrización inapropiada del espacio de Reinke con la consecuente disfonía persistente justifican su utilización. El procedimiento se puede realizar en forma bilateral cuidando que las incisiones en ambas cuerdas no tomen contacto. Es muy importante considerar que dado que el tejido succionado por el microdebridador no puede recuperarse cualquier leucoplaquia u otra lesión sospechosa de malignidad debe ser removida con técnica clásica y enviada a estudio anatomo-patológico.

\section{BIBLIOGRAFÍA}

1. Zeitels SM, Casiano RR, Gardner GM, Hogikyan ND, Koufman JA, Rosen CA. Management of common

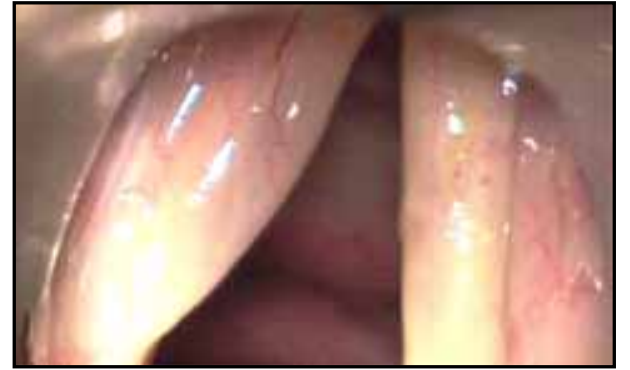

Figura 1. Imagen de la laringe antes de la cirugía.

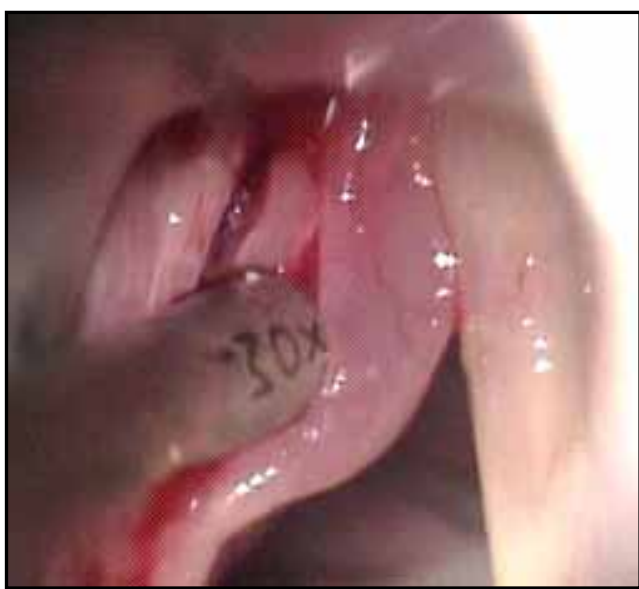

Figura 2. Succión del mixioma con microdebridador.

voice problems: Committee report. Otolaryngol Head Neck Surg. 2002; 126(4): 333-48.

2. SiUPSINSKIENE N, SkUmANIENE M. Phonatory characteristics following different endolaryngeal microsurgical techniques in Reinke's disease. Medicina 2002;38(10):982-9

3. Hirano M, Shin T, Morio M, et al. An improvement in surgical treatment for polypoid vocal cord: sucking technique. Otologia 1976; 22: 583-9.

4. Yates A, Dedo HH. Carbon dioxide laser enucleation of polypoid vocal coros. Laryngoscope 1984; 94: 731-6.

5. Dursun G, Ozgursoy OB, Kemal O, Coruh I. One-year follow-up results of combined use of $\mathrm{CO} 2$ laser and cold instrumentation for Reinke's edema surgery in professional voice users. Eur Arch Otorhinolaryngol. 2007; 264(9): 1027-32.

6. Druck G, Mauri M. Use of the microdebrider for Reinke's edema surgery. Laryngoscope 2000; 110: 2114-6. 